\title{
Millikelvin Cryogenics at Fermilab
}

\section{Matt Hollister, APS-TD Cryogenic Engineering}

\section{Introduction}

Millikelvin cryogenics is an emergent area for Fermilab with broad applications in particle detection for the cosmic frontier and quantum information science.

Fermilab Cryogenic Engineering currently supports multiple experiments, including the SuperCDMS SNOLAB and ADMX dark matter experiments, along with several millikelvin test facilities. More programs are expected to come online in future.

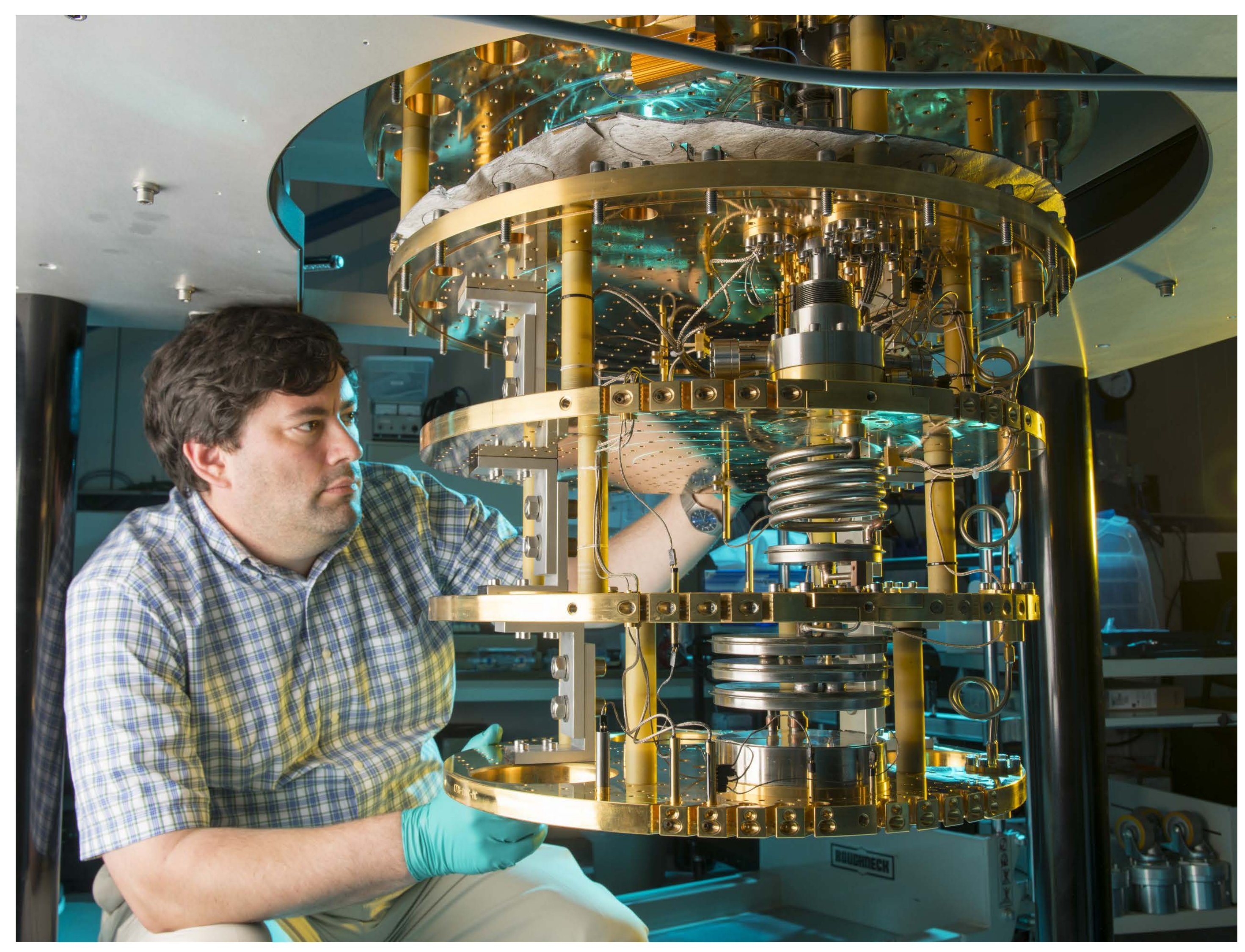

The interior of the SuperCDMS SNOLAB dilution refrigerator Constructed by Leiden Cryogenics, the fridge is the currently the most powerful commercial refrigerator model available, providing over 2 $\mathrm{mW}$ at $100 \mathrm{mK}$ and a base temperature of $5 \mathrm{mK}$

\section{Challenges in Millikelvin Cryogenics}

Designing systems and experiments for $\mathrm{mK}$ cryogenic operation affords additional challenges compared to higher temperatures.

- Limited cooling capacity. Cooling powers at $100 \mathrm{mK}$ typically 5 to 6 orders of magnitude lower than available at $1 \mathrm{~K}$.

- Complex material choices. Since thermal conductivity varies approximately linearly with temperature, at millikelvin temperatures even copper must be used carefully for thermal links. Many common engineering materials will be superconducting, leading to unexpected thermal impedances and excess heat capacities.

- Experiment assembly. Common material joining techniques such as hard soldering can be problematic at millikelvin temperatures. Even bolted contacts that would be functional at liquid helium temperatures can present large thermal resistances at lower temperature due to increasing thermal boundary effects.

- Limited measurements of material properties. Available literature data for materials and components is sparse and often inconsistent.

\section{Millikelvin Facilities at Fermilab}

Three permanent millikelvin facilities are currently operational at Fermilab, supported in part by Engineers from the Cryogenics Sector. In addition, several smaller cryostats are available for quick turnaround, small-scale R\&D tests.

\section{Quantum Metrology Laboratory}

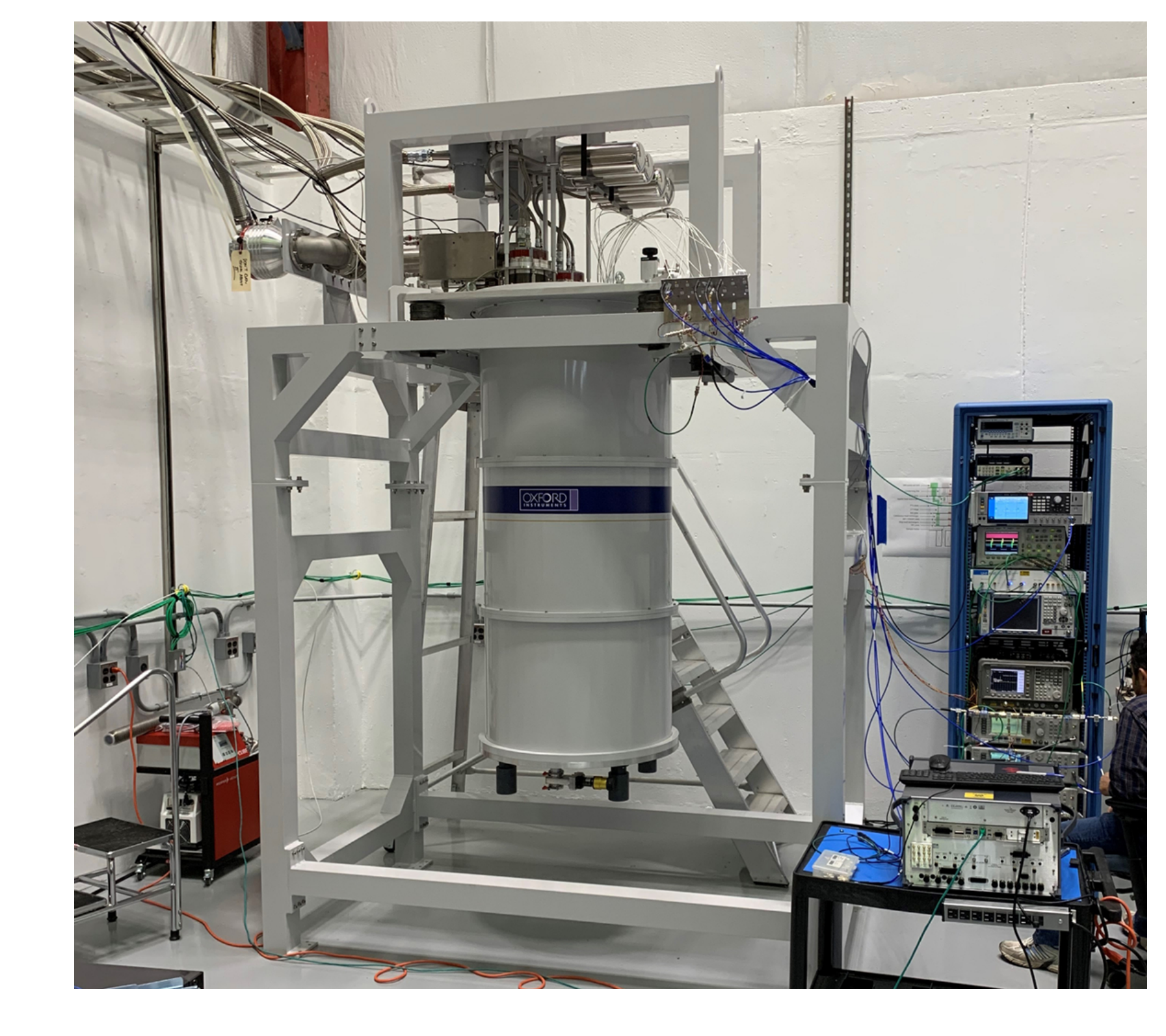

Quantum Metrology Laboratory dilution refrigerator from Oxford Instruments in Lab B.
- Located in Lab B, the Quantum Metrology facility includes a large-frame refrigerator providing $12 \mu \mathrm{W}$ at $20 \mathrm{mK}$.

- Can be operated with a 14 Tesla, $90 \mathrm{~mm}$ bore superconducting magnet with active shielding.

- Currently used for axion dark matter detector development.

\section{Quantum Computing Laboratory}

- The ICB Quantum Computing Laboratory includes two refrigerators from BlueFors providing $30 \mu \mathrm{W}$ at $20 \mathrm{mK}$.

- Large experimental volumes, up to $500 \mathrm{~mm}$ diameter and $1.4 \mathrm{~m}$ high.

- Currently utilized for highQ superconducting resonator development.

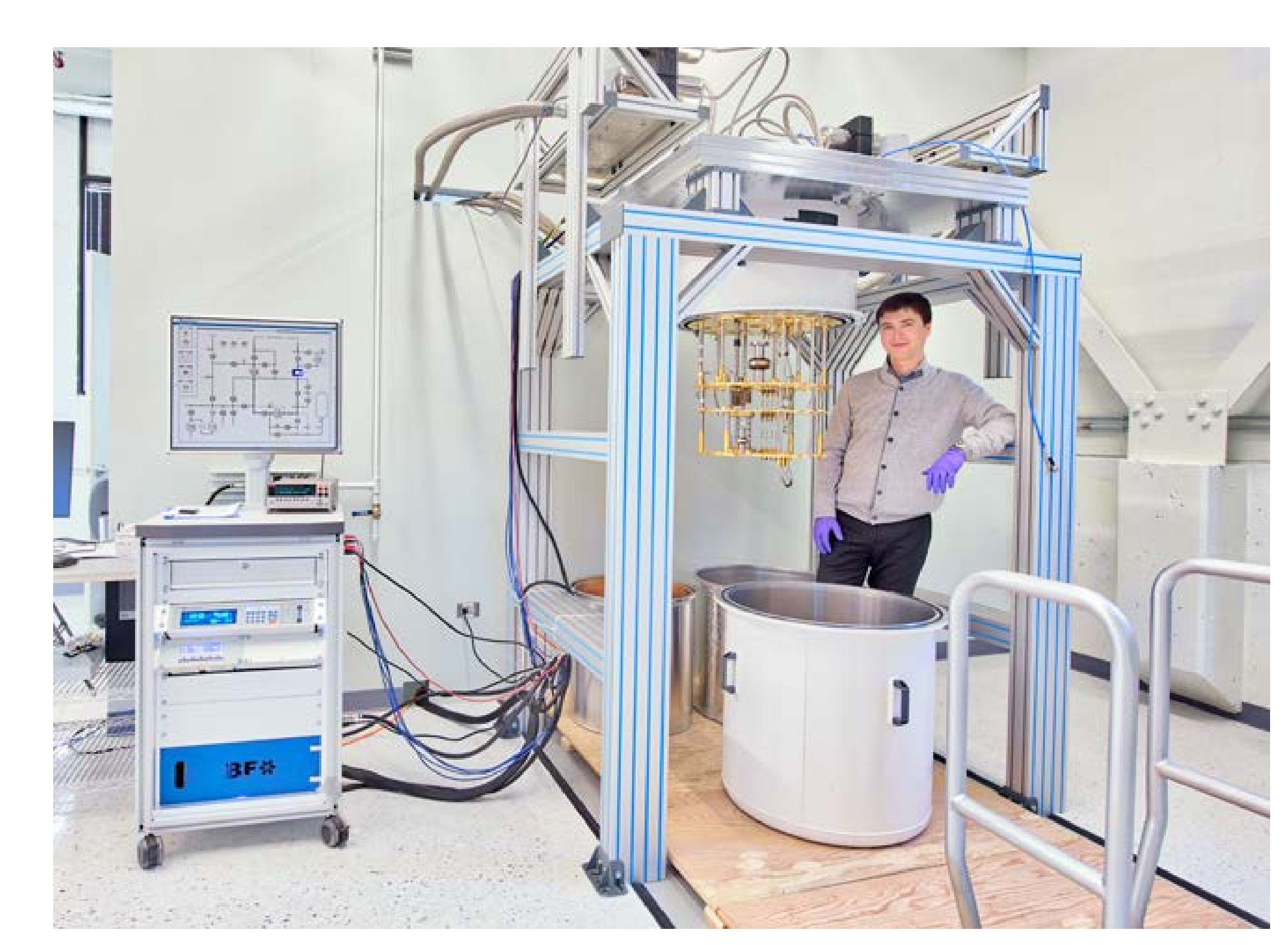

One of two BlueFors XLD dilution refrigerators in the ICB Quantum Computing Laboratory.

\section{Northwestern Experimental Underground Site (NEXUS)}

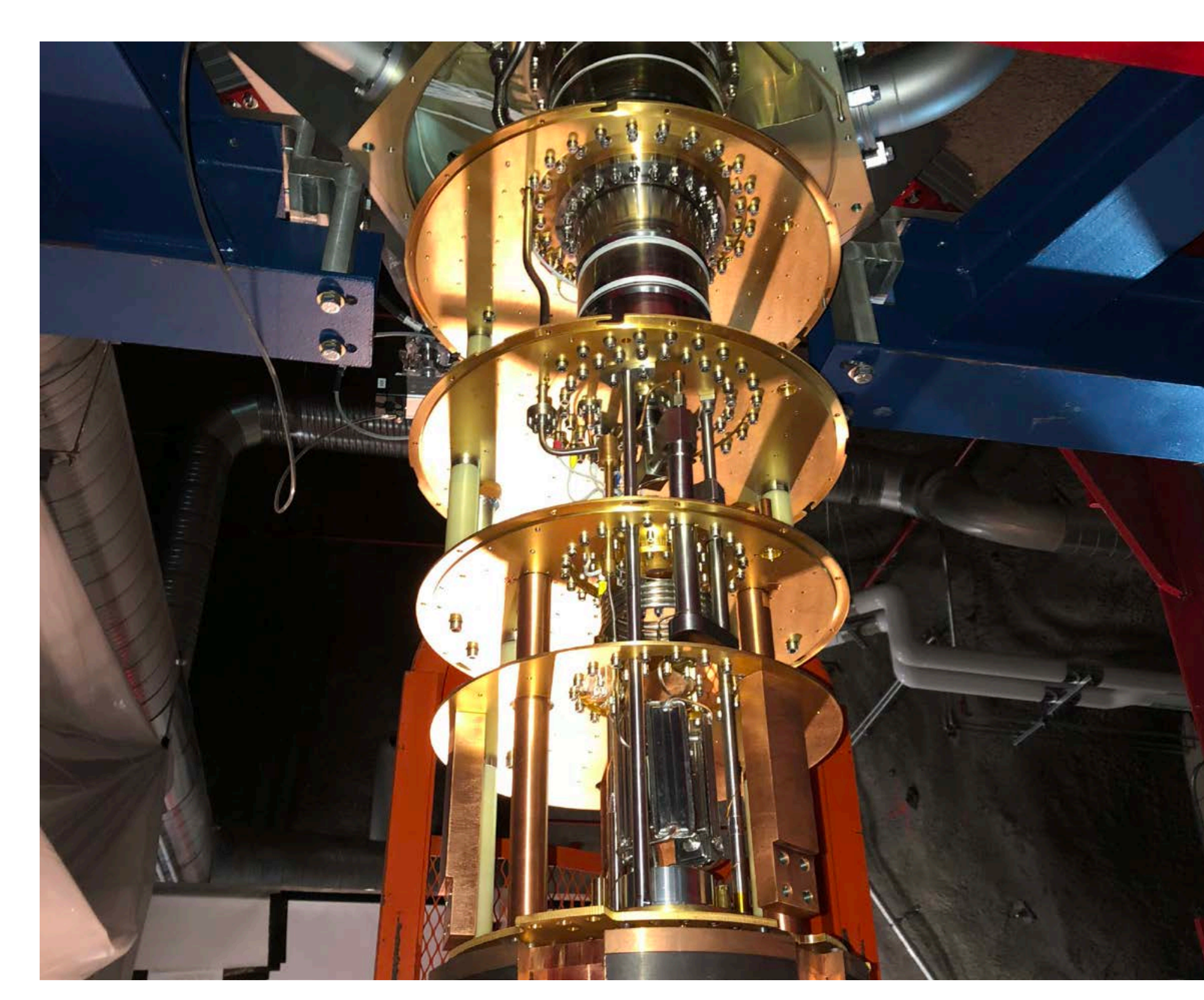

Interior of the NEXUS refrigerator underground in MINOS. The fridge features the unique "Ultraquiet" cryocooler system from CryoConcepts that mechanically decouples the pulse tube cooler from the fridge to provide an extremely low vibration fridge.
- NEXUS places a dilution refrigerator 300 feet underground adjacent to the MINOS hall.

- The rock overburden shields the fridge from much of the cosmic ray flux at the surface.

- NEXUS is currently used for calibration of the SuperCDMS SNOLAB detectors, along with other low-background detector and qubit R\&D projects. 